\title{
Single Stage Nasal Reconstruction in a Near Total Nasal Defect
}

\author{
Vimalendu Brajesh ${ }^{1} \quad$ Aditya Aggarwal ${ }^{1} \quad$ Sukhdeep Singh ${ }^{1} \quad$ Vishal Vora ${ }^{1}$ Kanika Rana \\ ${ }^{1}$ Department of Plastic, Aesthetic and Reconstructive Surgery \\ Medanta the Medicity, Gurgaon, Haryana, India \\ 2Department of Head and Neck Surgery Medanta the Medicity, \\ Gurgaon, Haryana, India

\begin{abstract}
Address for correspondence VimalenduBrajesh, MCh (Plastic surgery), Department of Plastic, Aesthetic and Reconstructive Surgery Medanta the Medicity, Sector- 38, Gurgaon, Haryana, 122001, India (e-mail: drvbrajesh@yahoo.co.in).
\end{abstract}

Indian J Plast Surg:2020;53:431-434

\begin{abstract}
Keywords

- near total nasal defect

- Radial artery forearm flap

- single stage nasal reconstruction

Reconstruction of nasal defect is difficult and challenging. A full-thickness defect of nose requires reconstruction of thin inner lining, middle skeletal (bony/cartilaginous) support, and outer skin layer cover. Large full-thickness defects of nose require complex multistage reconstruction to achieve good functional and aesthetic result. We present here a case of 12-year-old boy, a known case of xeroderma pigmentosa, who underwent wide local excision for squamous cell carcinoma of the nose, leaving a near total defect of the external nose. The reconstruction was done with a suprafascial, thin radial artery forearm free-flap for the external cover as well as the inner lining along with the septal cartilage graft for skeletal support in a single stage.
\end{abstract}

\section{Introduction}

Reconstruction of nasal defects is extremely challenging. A full-thickness nasal defect requires reconstruction of the thin inner mucosal lining, middle skeletal (bony/cartilaginous) support, and outer skin cover. In smaller defects, reconstruction can be done with local or regional flaps, but in larger defects, complex multistage reconstruction is needed to achieve good functional and aesthetic outcome.

\section{Case Report}

A 12-year-old male from Yemen, a known case of xeroderma pigmentosa, presented with a lesion over the left alar region for the last 3 to 4 months. The lesional biopsy reported a poorly differentiated squamous cell carcinoma. There was a similar lesion on the right ala of the nose (-Figs. 1-3). Large full-thickness defects of nose are usually corrected using adipofacial radial artery-free flap for inner lining and a forehead flap for outer skin cover. However, in this case, a single-stage reconstruction was planned with radial artery-free flap.

\section{Surgical Procedure and Planning}

The reconstructive and oncosurgical teams discussed the histopathology report and planned a wide local excision with $1 \mathrm{~cm}$ margin all around. The defect, after the wide local excision, can be seen in $\mathbf{- F i g s .} \mathbf{4}$ and $\mathbf{5}$.

To correct the defect, reconstruction of inner lining of both the alar regions, lining for the septal cartilage on both sides in the lower part, and the skin cover over the lower half of the nose, the collumella and the upper part of the philtrum, were needed ( - Figs. 6 and $\mathbf{7}$ ). Structural support was required for tip and columella, in order to maintain the nasal height and provide a definition and projection to the tip of the nose. To prevent the collapse of lateral walls, the reconstruction of upper lateral cartilages and alar rims was also needed. published online

December 30, 2020
DOI https://doi.org/

$10.1055 / \mathrm{s}-0040-1721858$ ISSN 0970-0358.
C2020. Association of Plastic Surgeons of India.

This is an open access article published by Thieme under the terms of the Creative Commons Attribution-NonDerivative-NonCommercial-License, permitting copying and reproduction so long as the original work is given appropriate credit. Contents may not be used for commercial purposes, or adapted, remixed, transformed or built upon. (https://creativecommons.org/licenses/by-nc-nd/4.0/).

Thieme Medical and Scientific Publishers Pvt. Ltd. A-12, 2nd Floor, Sector 2, Noida-201301 UP, India 


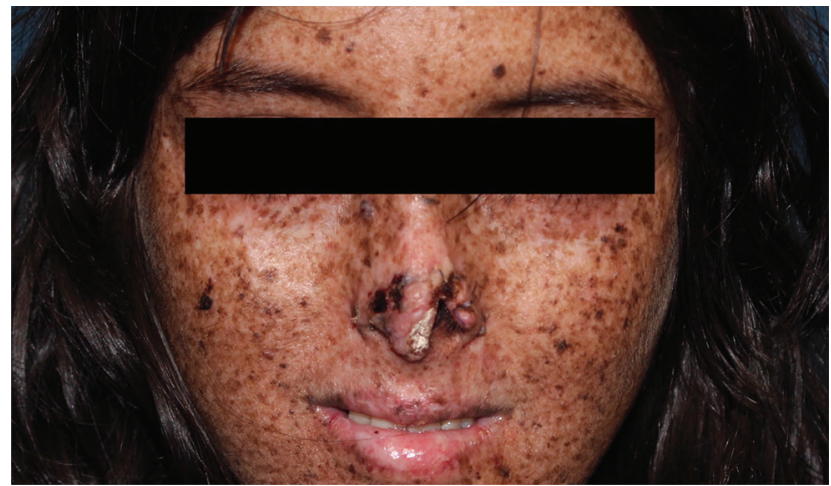

Fig. 1 Frontal view of lesion (defect shown is after biopsy).

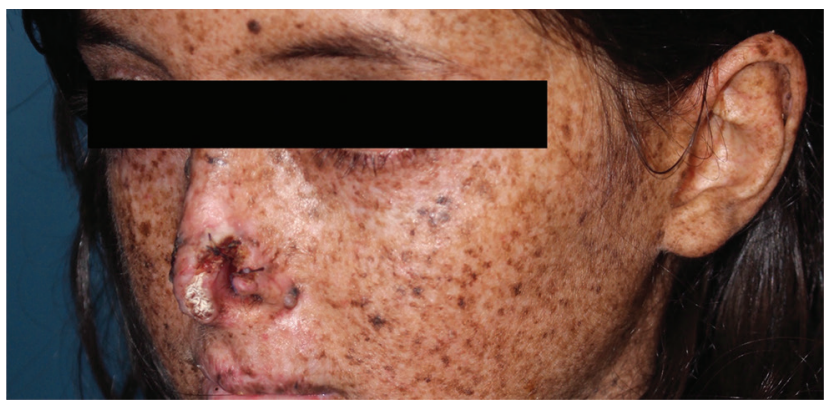

Fig. 2 Oblique view of lesion (defect shown is after biopsy).

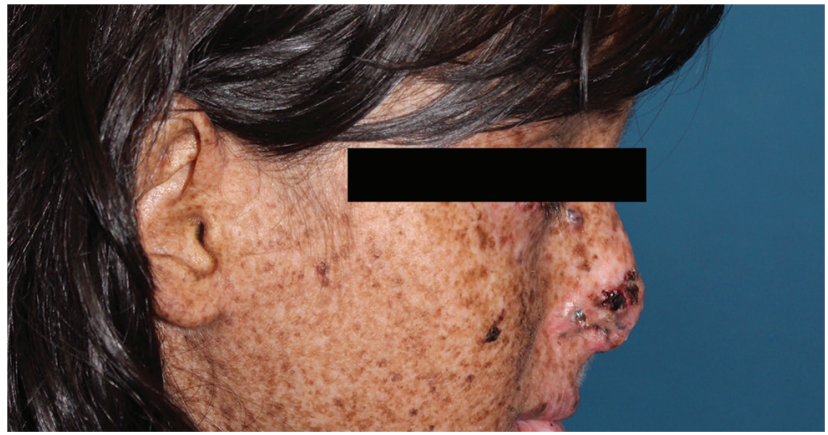

Fig. 3 Lateral view of lesion (defect shown is post biopsy).

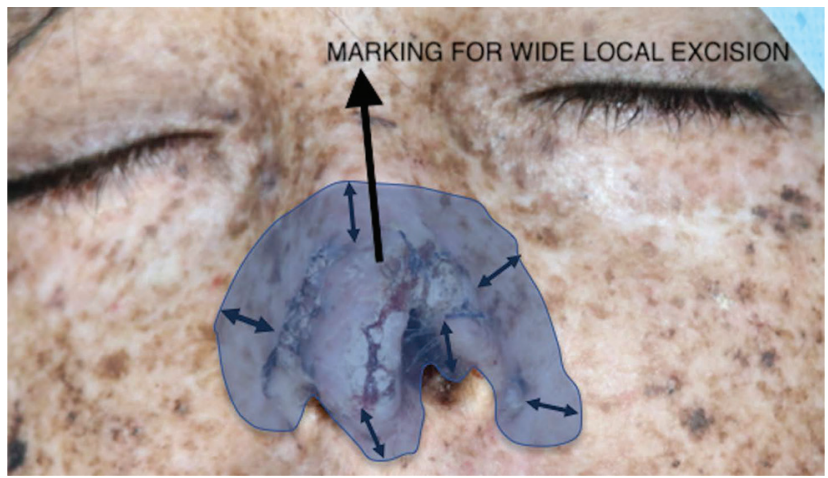

Fig. 4 Planned wide local excision with a margin of $1 \mathrm{~cm}$.

The cartilage graft needed for skeletal support was harvested from the residual septal cartilage. The harvested cartilage was used for the newly created collumellar strut, alar rim graft, and upper lateral cartilages ( - Fig. 8). A suprafascial radial artery forearm-free flap was harvested from the

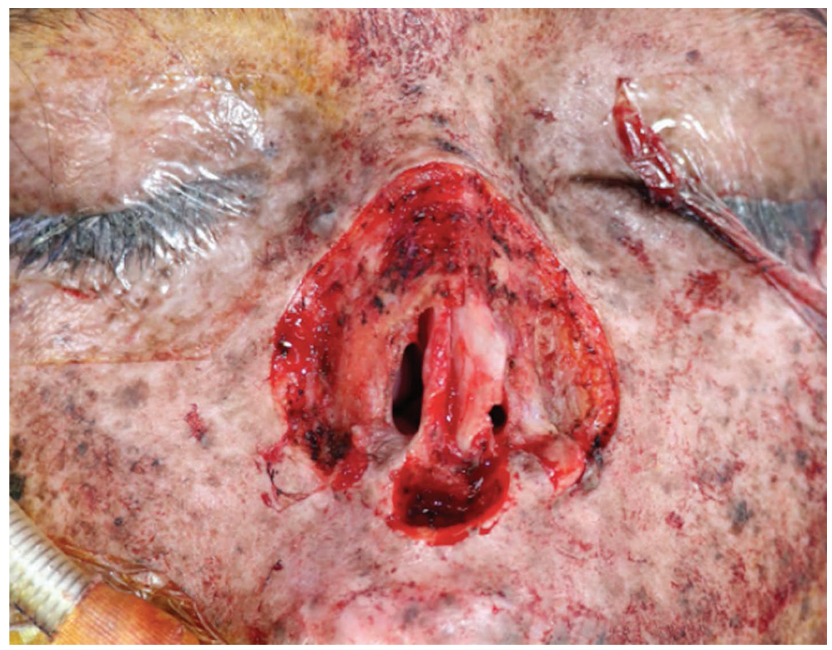

Fig. 5 Nasal defect after wide local excision (WLE).

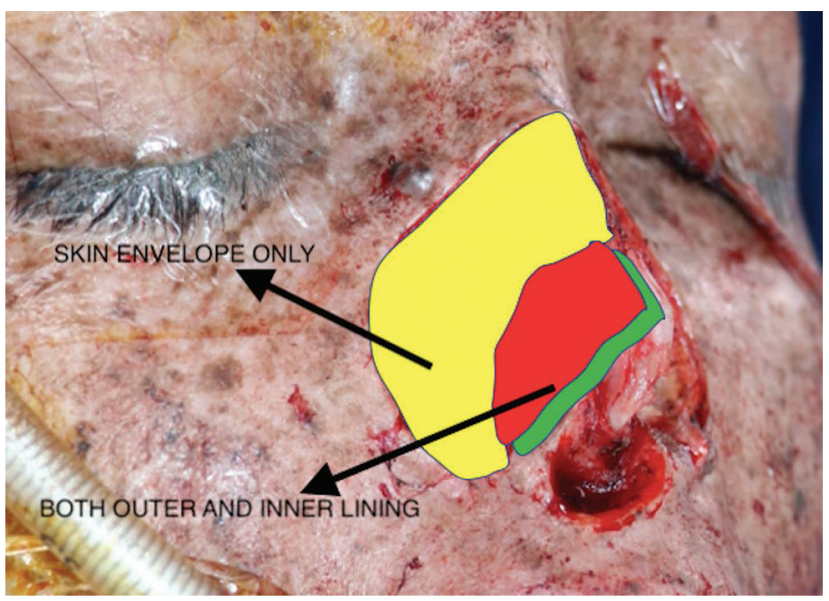

Fig. 6 Area of skin cover required for reconstruction right lateral nose and ala. Yellow-colored area represents outer skin envelope requirement over the lateral wall of the nose. Red- and green-colored area represents outer skin envelope and inner cover requirement, respectively, in the right alar region.

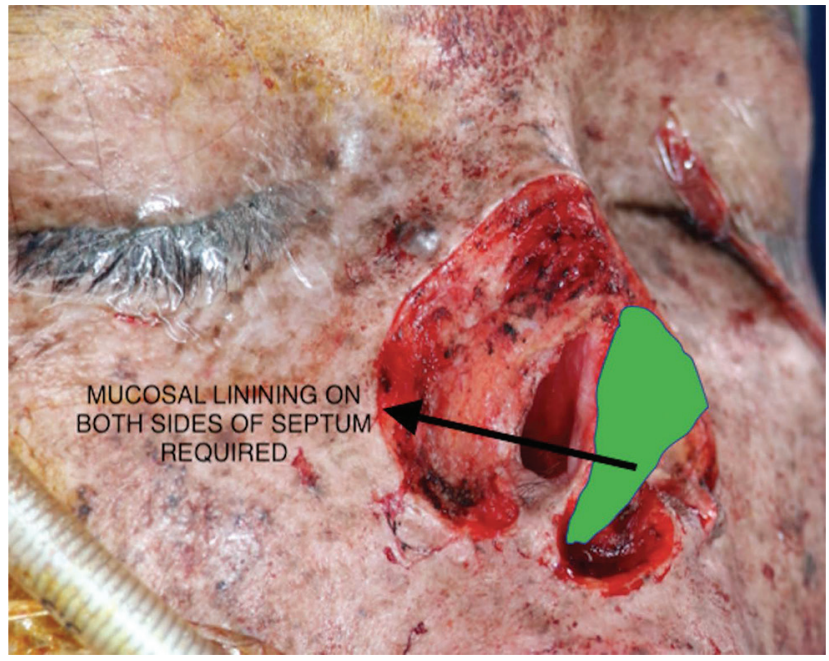

Fig. 7 Green-colored area represents the part of central septum, which needs to be covered by both the sides. 


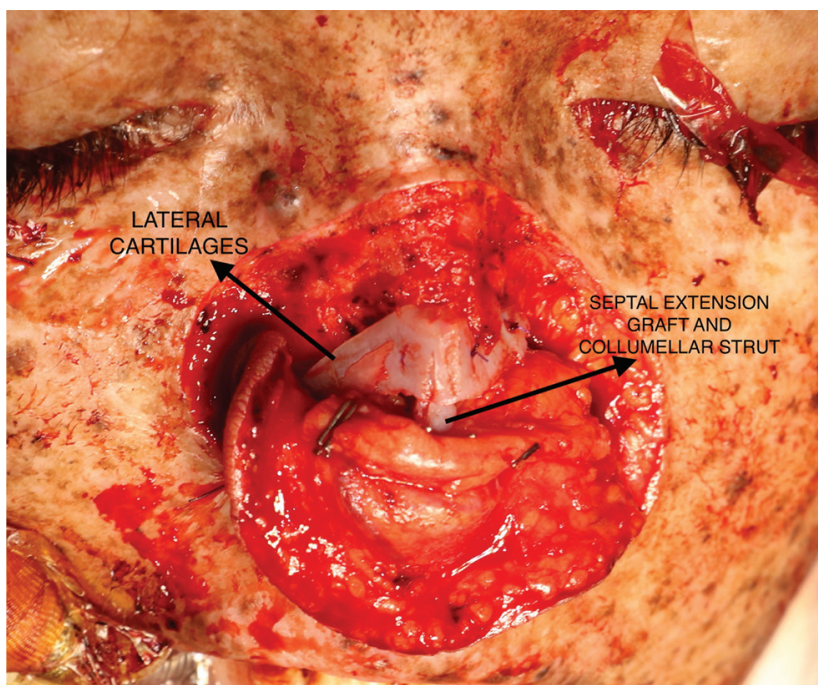

Fig. 8 The arrows represent the reconstructed cartilages (lateral cartilages, septal extension graft, and collumellar strut).

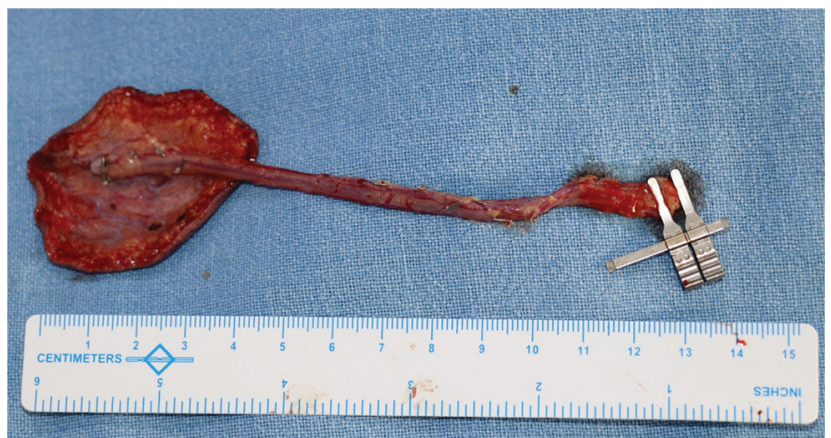

Fig. 9 Harvested radial artery forearm-free flap.

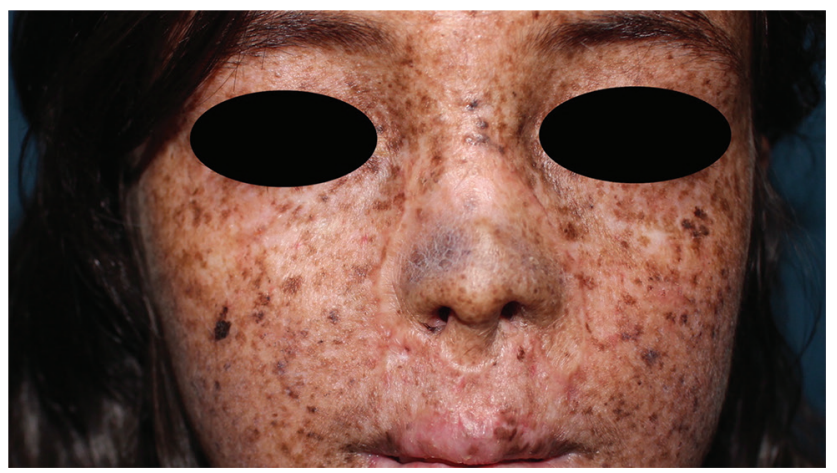

Fig. 10 Frontal view after reconstruction (4 months follow-up).

(nondominant) left upper limb ( - Fig. 9). As a first step, the inner lining was created by insetting the flap along the residual mucosal lining. It was further thinned to dermal fat level. The flap was then turned over to provide the skin cover. The pedicle was tunneled subcutaneously on the left side and anastomosis was done to the facial vessels in the submandibular region. The patient's postoperative course was uneventful, and he was discharged on postoperative day 5 with a well-settled flap. The postoperative result after 4 months can be seen in the picture (-Figs. 10 and $\mathbf{1 1}$ ).

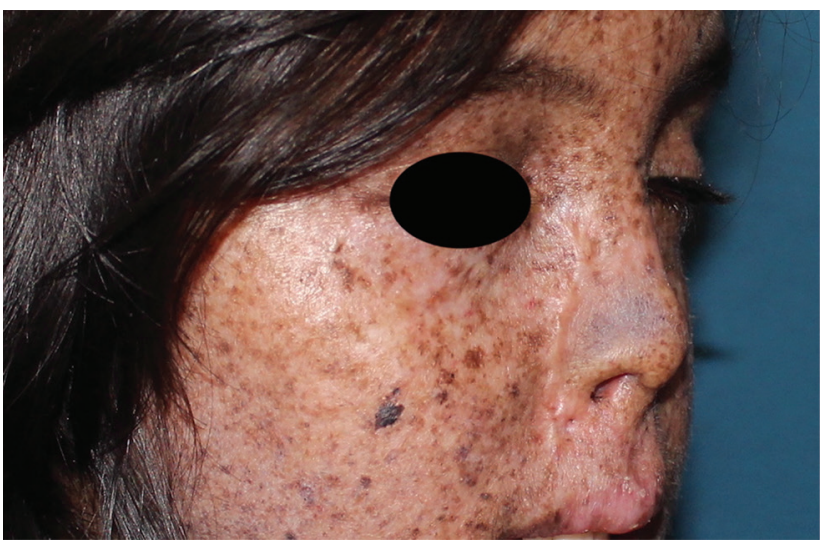

Fig. 11 Oblique view after reconstruction (4 months follow-up).

The use of nasal splint to maintain the long-term patency of the nostril is an important aspect for good functional outcome. ${ }^{1}$ A custom-made nasal splint with patent airway was given to the patient to be used after 1 month and continued till 4 months' postop.

\section{Discussion}

Xeroderma pigmentosa ${ }^{2}$ is rare autosomal recessive disease in which the DNA repair mechanism gets hampered. The skin becomes hypersensitive to ultraviolet (UV) radiation. There is high incidence of skin cancer and progressive neurological complications. Majority of patients suffer from squamous cell carcinoma at 10 to 15 years of age.

The nose is one of the most complex facial structures to reconstruct. Failure to restore the structural stability of the nose can be devastating to the patient. The most common causes of total/near total nasal defect presently are trauma or cancer resection. ${ }^{3}$ Reconstruction of a full-thickness nasal defect requires reconstruction of all the three layers of the nose.

For small defects, the adjacent tissue raised as local flaps can serve as a good reconstructive option. However, for large defects, free-tissue transfer is the only option. ${ }^{4}$ The creation of inner lining is the most complex, as it is a very specialized thin layer. Skin graft lining is an option for small defects, but they contract unpredictably, and at the same time preclude cartilage grafting. ${ }^{4}$ Lining provided by skin flaps are often bulky and obstruct the airway. ${ }^{1}$ Secondary thinning is most often required to restore the patency of the nostrils. Radial artery-free flaps can be primarily thinned to give a good inner lining without any vascular compromise. The possibility of postoperative scarring and subsequent contracture should be accounted for at the time of surgery. The cartilaginous structural support created during our single-stage repair will ensure this.

The skin envelope may be reconstructed with skin grafts or a variety of flaps including nasolabial flaps, forehead flaps, and free flaps. Forehead flap is inherently a two-staged procedure, which involves resurfacing of the defect in the first stage and division of pedicle and inset in the second 
stage. ${ }^{4}$ For all practical reasons, a third/fourth stage is always needed for flap thinning, realignment of the cartilages or placement of hitch stitches to create the creases and natural depression, and an acceptable aesthetic outcome.

For reconstruction of large full-thickness defects of the nose, placement of tissue expander, prefabrication of forehead flap, ${ }^{5}$ and prefabricated radial artery-free flap ${ }^{6}$ are alternative options, but they are not useful when immediate reconstruction is required like in wide local excision of squamous cell carcinoma of the nose. A combination of radial artery-free flap and forehead flap is the only option in such cases. In this case, we preferred the radial artery-free flap, as the forehead is an exposed area predisposed to cancerous changes due to xeroderma Pigmentosa.

Skeletal support to replicate the cartilaginous framework of the nose is an important step to prevent the collapse of the nose under the weight of soft-tissue cover and withstand the contractile forces of the scar tissue. Cartilage or bony framework for skeletal support are either given as a part of prefabrication or inserted later as a staged procedure. Providing the inner lining as well as outer skin cover with the same flap allows to create a closed environment where cartilage framework can be placed in the same sitting. Microvascular-free flap also gives the liberty of placing the cartilages in the subdermal plane by creating a tunnel between the subcutaneous tissue and the dermis without hampering the blood circulation to the flap, as radial forearm flap gives multiple perforators along its axial course. The alar rim cartilage, which is very important to prevent the notching and retraction of the ala, needs secondary adjustment in all cases where multistage reconstruction is done. We placed the rim cartilage, at exact location, in the first step itself, thus allowing us the opportunity of a single-stage repair. The anatomical integrity of the alar rim can be noticed in the postoperative photographs of the patients.

The alar facial sulcus is an important topographical region, because it represents the transition zone of three cosmetic units: the nasal base, the medial aspect of cheek and the upper lip. ${ }^{7}$ Creation of sulcus needs placement of hitch stiches, which is usually done as a second-stage procedure to prevent vascular compromise of pedicle flaps. In the present case, we placed the stitches in the same sitting. Interrupted stitches from dermis of the flap to the underlying cheek soft-tissue to create a groove was done in the first stage itself. Free flaps in our opinion better tolerate hitch stitches compared with the pedicled flaps, as the free flap, in particular the radial artery flap, has an axial vessel running along the course of the entire flap and therefore better perfusing the flap as compared with the pedicled flaps, where the distal most tip of the flap is the most compromised and any hitch sutures can further compromise the flap.

\section{Conclusion}

The suprafascial radial artery forearm-free flap can be used for a single-stage reconstruction of large nasal defects, providing excellent external as well as internal lining to give good functional and good aesthetic outcome.

\section{Conflicts of Interest}

None declared.

\section{References}

1 Burget GC, Walton RL. Optimal use of microvascular free flaps, cartilage grafts, and a paramedian forehead flap for aesthetic reconstruction of the nose and adjacent facial units. Plast Reconstr Surg 2007;120(5):1171-1207, discussion 1208-1216

2 Naik SM, Shenoy AM, Nanjundappa A, et al. Cutaneous malignancies in xeroderma pigmentosum: earlier management improves survival. Indian J Otolaryngol Head Neck Surg 2013;65(2):162-167

3 Cannady SB, Cook TA, Wax MK. The total nasal defect and reconstruction. Facial Plast Surg Clin North Am 2009;17(2):189-201

4 Menick FJ. Practical details of nasal reconstruction. Plast Reconstr Surg 2013;131(4):613e-630e

5 Mutaf M, Ustuner ET, Celebioglu S, Kocer U, Sensoz O. Tissue expansion-assisted prefabrication of the forehead flap for nasal reconstruction. Ann Plast Surg 1995;34(5):478-484, discussion 485-487

6 Ahcan U, Didanovic V, Porcnik A. A unique method for total nasal defect reconstruction - prefabricated innervated osteocutaneous radial forearm free flap. Case Reports Plast Surg Hand Surg 2019;6(1):11-19

7 Baker SR, Johnson TM, Nelson BR. The importance of maintaining the alar-facial sulcus in nasal reconstruction. Arch Otolaryngol Head Neck Surg 1995;121(6):617-622 